\title{
Pulse Shape Dependence of Vapor Shielding Efficiencies During Transient Heat Loads
}

\author{
Kenzo IBANO, Yoshio UEDA and Tomonori TAKIZUKA \\ Graduate School of Engineering, Osaka University, Suita 565-0871, Japan
}

(Received 21 May 2021 / Accepted 16 July 2021)

\begin{abstract}
Erosion of first walls in tokamak fusion reactors due to transient heat loads during ELM and disruptions is a major concern and needs to be predicted. Studies have shown that the erosion amount is strongly dependent on the total energy density and duration of a transient heat pulse. Recently, it was pointed out that the erosion amount is also dependent on the pulse shape [J.H. Yu et al., Nucl. Fusion 55, 093027 (2015), and D. Motoi et al., Fusion Eng. Des. 165, 112209 (2021)]. Meanwhile, it is predicted that the erosion during the transient heat loads can be suppressed by vapor shieldings, and the efficiency of the vapor shielding may differ between the pulse shapes. Thus, in this study, we investigate the pulse shape dependence of the vapor shielding effect by a particle-in-cell based simulation code, PIXY. Two types of square shapes and three types of triangular shapes are examined. Among the triangular shapes, it is found that the vapor shielding is effective especially in "Negative Ramp" triangular shape, where the peak heat flux comes first.
\end{abstract}

(C) 2021 The Japan Society of Plasma Science and Nuclear Fusion Research

Keywords: vapor shielding, particle-in-cell simulation, transient heat load, plasma surface interaction

DOI: $10.1585 /$ pfr.16.1405092

\section{Introduction}

A major concern in the operation of ITER and DEMO is the erosion of the plasma facing wall. We need to correctly predict the effects of plasma heat and particle fluxes into the wall surface. In the steady-state operation, heat flux exceeding several $\mathrm{MW} / \mathrm{m}^{2}$ will highly increase the surface temperature and particles flux of energy exceeding several tens of eV will cause substantial sputtering. Meanwhile, active cooling or plasma detachment can handle the heat loads [1,2]. Even for the substantial sputtering condition, the prompt redeposition will mitigate the erosions if the ionization mean-free path of the wall ejected particles is sufficiently shorter than its gyro radius [3]. We are having solutions for the surface erosion caused by the steady state loads as described.

Another concern is the transient loads caused by plasma instability such as ELMs and disruptions [1,2]. In the transient loads, significant heat loads reach the surface, the metallic surface melts for a short period of time (a few $\mathrm{ms}$ ), and massive evaporation or ablation occurs. Resulting molten layer is transported by electromagnetic forces and re-solidified $[4,5]$. The modified uneven surface will induce arcing, and a bridging between components, which increases mechanical stress or leads to electrical shorts.

Thus, erosion caused by the transient loads should be well investigated. High heat flux tests have been conducted by electron beam devices [6-8] and plasma guns [9-11]. These studies revealed that the erosion amounts can be summarized in the heat flux factor $F_{\mathrm{HF}}=\varepsilon_{\perp} \tau^{-0.5}$ where $\varepsilon_{\perp}$ is the energy density into the target surface and $\tau$ is the heat pulse duration $[1,12]$.

On the other hands, recent experimental studies pointed out an importance of pulse shapes to the erosion prediction during the transient loads $[13,14]$. According to a previous study [14], it was found that the pulse with its peak heat flux coming first brought the smaller erosion. In the cases of disruption in tokamaks, the peak heat flux is expected to come first, thus the erosion may be smaller than the predictions assuming square pulse shapes.

In addition, the vapor shielding effect is expected to be an inherent erosion suppression effect to the transient heat load. When a large amount of vapor is ejected from the plasma-facing surface, the vapor shielding reduces the incoming plasma energy due to the interaction between the vapor and the plasma. Numbers of studies have been conducted for the vapor shielding by experiments $[15,16]$ and simulations [17-19], and erosion suppression effects of vapor shielding were confirmed. However, these studies did not carefully consider the impact of the pulse shape on the vapor shielding effects. There is a large possibility of the vapor shielding efficiency to be dependent on the timing of vapor emissions.

Thus, in this study, we evaluate pulse shape dependence of the vapor shielding efficiencies using our particlein-cell (PIC) based vapor shielding simulation code, PIXY, in a one-dimensional system. Calculations are performed for the transient loads of deuterium (D) plasma into a beryllium (Be) first wall considering the ITER wall [20]. 


\section{PIXY Simulation}

PIXY simulates plasma-surface interactions using a PIC plasma model and a wall heat transfer model. In the PIC model, multiple plasmas and impurities can be treated. Coulomb collisions are simulated by a Monte-Carlo binary collision model $[21,22]$. Ionization/recombination reactions, radiation power losses and elastic collision with neutral particles are calculated based on the OPEN-ADAS database [23].

A unique point of the PIXY is its capability for emitted vapor particles. In the vapor shielding simulation, the amount of vapor emission varies greatly with time. Thus, a weighted PIC model is employed to change the weight of each super-particle. Using the weighted PIC, the variety of vapor particle flux can be simulated with a reasonable statistical accuracy. The plasma simulation by the PIC model calculates incoming particle energy, angle of incident, and heat flux to the wall. In the wall model, sputtering is calculated using Eckstein's sputtering rate as a function of the energy and the angle of incidence of the incoming plasma particles. In the heat transfer calculation, the heat flux value from the PIC model is used as a boundary condition of the wall surface. A $10 \mathrm{~mm}$ thick Be wall and a $7 \mathrm{~mm}$ thick copper pipe are assumed. The back-side boundary of the copper pipe is assumed as water cooling by $343 \mathrm{~K}$ in the present study. The temperature distribution inside the wall is calculated and the surface temperature is obtained. When the surface temperature is below the boiling point, vapor is emitted according to the vapor pressure of the material. If the surface temperature exceeds the boiling point, we assume ablation, and the vapor corresponding the thickness of surface layers exceeding the boiling point is emitted. The erosion amount is calculated from the sputtering and vaporization amounts. The erosion occurs at each time step reduces the wall thickness, then the spatial wall mesh was re-meshed as the erosion occurs. Further details are found in $[18,19]$.

We investigate the pulse shape dependence of the vapor shielding effect. For the same "input" energy density $\varepsilon_{\text {in }}$, we set a "Full Width Half Maximum" square pulse with duration $\tau_{0}$ and heat flux $q_{0}\left(\varepsilon_{\text {in }}=q_{0} \tau_{0}\right)$. The $\tau_{0}$ value is set $1 \mathrm{~ms}$ and $q_{0}$ is varied up to $3 \mathrm{GW} / \mathrm{m}^{2}$ in the present study. To find the pulse height effect, "Half Width Full Maximum" square pulse with $q=2 q_{0}$ and half duration $\tau=\tau_{0} / 2$ is chosen. This pulse has larger "input" heat flux factor $F_{\mathrm{HF}}$ by $\sqrt{2}$ than the "Full Width Half Maximum" case.

In order to study the pulse shape effect, we use triangular pulses with the peak heat flux $q_{\mathrm{p}}=2 q_{0}$ and the duration $\tau=\tau_{0}$. The input energy density $\varepsilon_{\text {in }}$ and averaged heat flux $\langle\mathrm{q}\rangle=\varepsilon_{\text {in }} / \tau$ are the same as those of "Full Width Half Maximum" case, and $F_{\mathrm{HF}}$ value is also the same. Because there is a large possibility of the vapor shielding efficiency to be dependent on the timing of vapor emission, three types of triangular pulse with the heat-flux peak positions different each other are examined: (1) "Negative ramp" with a peak at the beginning, (2) "Positive ramp" with a peak at the end, and (3) "Symmetric triangle" with a peak at the middle of the pulse. These definitions are based on Yu's paper [24].

Pulse shapes at the actual tokamak devices depends on the multiple conditions of instabilities; for ELMs, the pedestal plasma collapse and the edge transport, for VDEs, the current quench and the halo current. In ITER, the expected rise time of ELM is $220-260 \mu$ s, and the decay time will be 2 - 4 times the rise time. Thus, the pulse shape will be somewhere between "Symmetric triangle" and "Negative ramp". Meanwhile, the pulse shape of VDEs is expected to be "Positive ramp" like shape [25].

In this paper, we focus on the effect of heat flux pulse shape. We need to emphasize here, that the plasma parameters, densities and temperatures, are also time dependent.

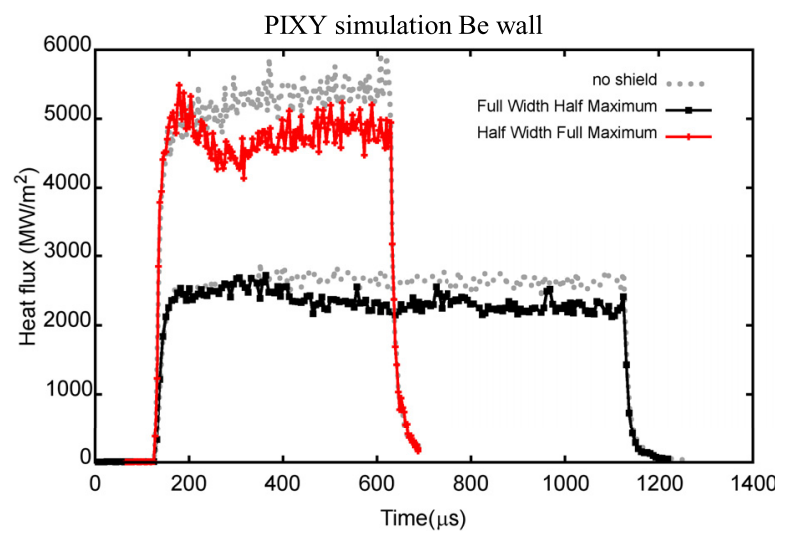

Fig. 1(a) Temporal development of heat flux for square pulses, "Full Width Half Maximum" and "Half Width Full Maximum". Solid lines show results with vapor shie lding and dashed lines show results without vapor shielding.

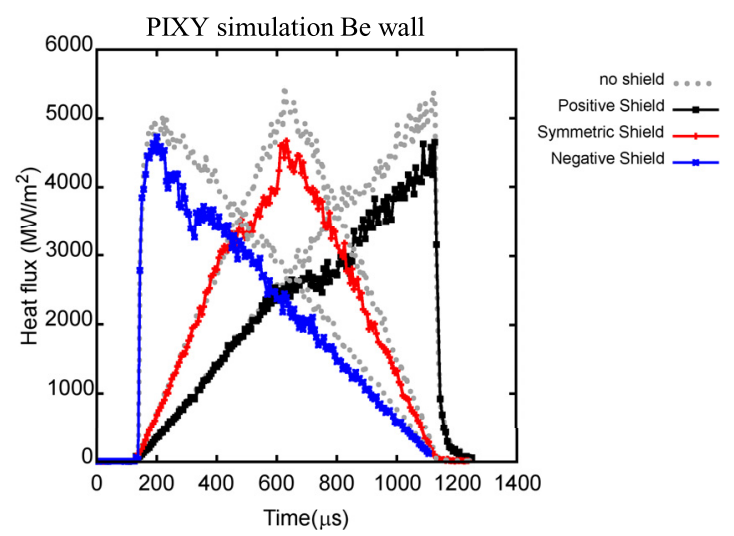

Fig. 1(b) Temporal development of heat flux for triangular pulse shapes, "Negative ramp" (blue), "Symmetric triangle" (red), and "Positive ramp" (black). Solid lines show results with vapor shielding and dashed lines show results without vapor shielding. 
Predictions of these parameters are challenging but should be studied in a near future.

We start the simulation of a background steady-state D plasma (electron density $n_{\mathrm{e}} \approx 10^{18} \mathrm{~m}^{-3}$ and plasma temperature $T \approx 200 \mathrm{eV}$ ) in a magnetic field injecting obliquely to the target with incident angle of $6^{\circ}$. The onedimensional system size is about $0.3 \mathrm{~m}$ with mesh number $\sim 10^{3}$. Afterwards, high temperature plasma was transiently injected into the upstream region. The injected plasma is adjusted sequentially to control the pulse shape of the transient loads. As shown in Fig. 1, the present PIC model successfully realizes the transient heat flux with various pulse shapes. The number of weighted super particles is $\sim 10^{5}$ in the steady state and is increased up to $\sim 10^{6}$ by injected hot plasma and emitted $\mathrm{Be}$ for the high vapor emission case.

\section{Results and Discussions}

\subsection{Heat flux reduction by vapor shielding}

Simulations are carried out by switching situations with and without vapor shielding. For the situation without shielding, Be emission amount is counted in accordance with the wall temperature, but Be super particles are not at all supplied to the system. Such technique is an advantage of the numerical simulation to clarify the vapor shielding effect. Figure 1 shows the temporal development of the heat flux to the target $q_{\perp}$ for "Full Width Half Maximum" and "Half Width Full Maximum" square-pulse cases with $q_{0}=2.5 \mathrm{GW} / \mathrm{m}^{2}$, where solid lines represent the situation with vapor shielding and dashed lines to the situation without vapor shielding. It is clearly seen the reduction of heat flux to the target by the vapor shielding. Simulation results of the heat flux reduction for the triangular heat pulses with $q_{\mathrm{p}}=2 q_{0}=5 \mathrm{GW} / \mathrm{m}^{2}$ are shown in Fig. 1 (b). These reductions were mainly achieved by the radiation cooling of electrons and the collisions between D ions and Be neutrals.

The time profiles of the wall surface temperature in these calculations are shown in Fig. 2, where the temperature is still below the boiling point of Be, $3243 \mathrm{~K}$. Between different pulse shapes, there is a difference in the maximum temperatures reached during the transient heat flux. This is partly because the pulse duration of the heat flux is shorter than the thermal diffusion. If the peak heat flux come later, such as "Positive Ramp", the peak heat flux is reaching while the heat is already stored on the surface. Therefore, the later the peak heat flux arrives in the pulse shape, the higher the maximum temperature reached. This tendency has also been confirmed in experiments [14,24].

When the vapor shielding is considered, we find reduced surface temperatures in each pulse shape.

\subsection{Erosion thickness}

Calculations are repeated with different energy densities, and the calculated erosion amounts are summarized

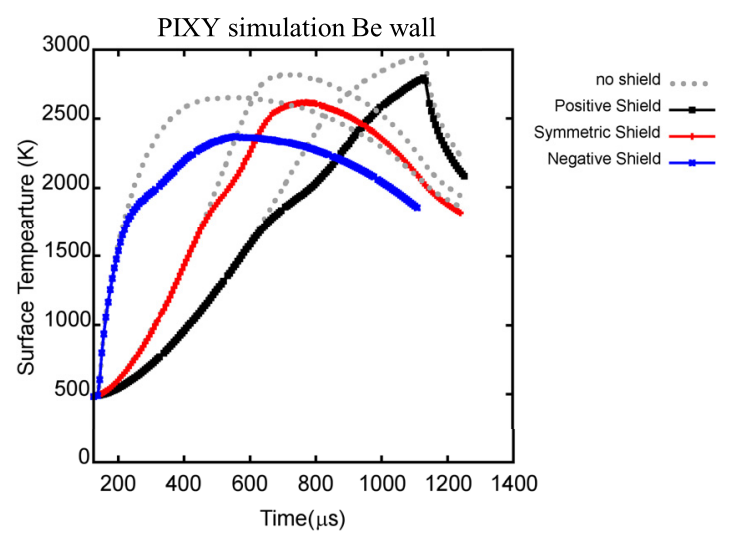

Fig. 2 Temporal development of surface temperature under the heat flux shown in Fig. 1. Solid and dashed lines represent results with and without vapor shielding, respectively.

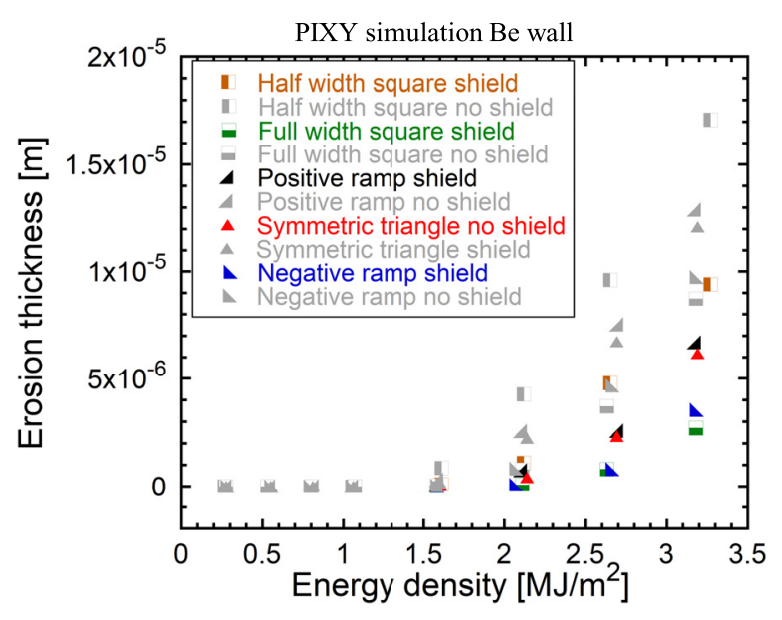

Fig. 3 Calculated wall erosion by a transient load as a function of energy density. Colored plots show results with vapor shielding and uncolored plots show results without vapor shielding.

in Fig. 3. Here, the erosions are mainly due to evaporation. The uncolored plots show the results without the vapor shielding, and the colored plots show the results with the vapor shielding. All the results of the triangular shapes were between results of the "Half Width Full Maximum" and the "Full Width Half Maximum" square pulse shapes. Among the triangular pulses, the "Positive Ramp" shows the largest erosion. This result was also inferred from the difference in the maximum temperature shown in Fig. 3. This simulation results by PIXY are qualitatively consistent with the experimental results taken by the authors (Fig. 4). Note that the experimental material was not Be but $\mathrm{W}$ (tungsten) and the heat source was intense Nd:YAG laser with $\sim 5 \mathrm{~ms}$ pulse width [14]. The erosion amounts were measured by the mass loss after 15 laser shots. The conversion from Ref. [14] to Fig. 5 are as follows; The energy plotted in Ref. [14] is labeled as "energy per shot [J]", 


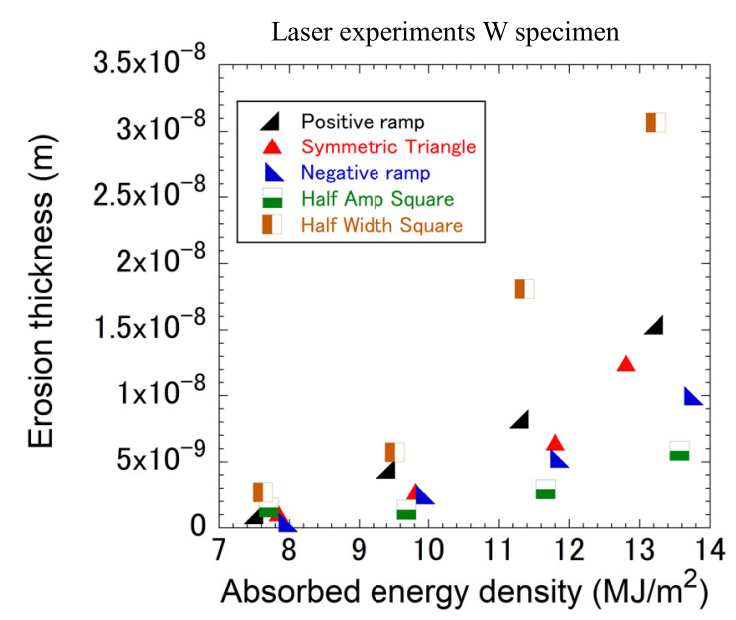

Fig. 4 Tungsten erosion thickness measured by Nd: YAG laser irradiations with different pulse shapes. (reproduced from Ref. [14])

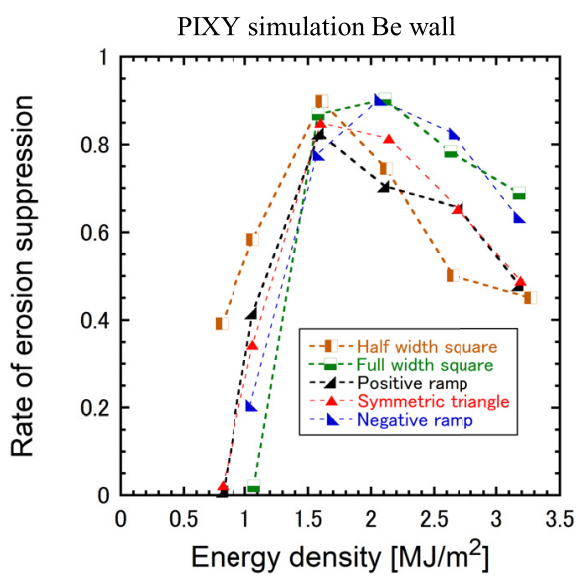

Fig. 5 Rate of erosion suppression by vapor shielding for various pulse shapes and as a function of energy density.

however the unit is actually in " $\left[\mathrm{MJ} / \mathrm{m}^{2}\right]$ ". The mass erosion of $0.1 \mathrm{mg}$ (W mass density $=19.3 \mathrm{~g} / \mathrm{cm}^{3}$ ) from this area corresponds to the thickness of $1.8 \times 10^{-7} \mathrm{~m}$. The mass erosion plotted in Ref. [14] is labeled as "erosion per shot", however this erosion amount is actually integrated erosion of 15 laser shots. Thus, the $0.1 \mathrm{mg}$ mass loss corresponds to $1.2 \times 10^{-8} \mathrm{~m}$. This experimental finding also shows that the erosions of triangular pulses are between the square pulses, and the largest erosion appears at the "Positive Ramp" cases. In the experiments, deformations of surface during the pulse may influence the results, but the main cause of this pulse shape dependence can be explained the maximum temperature discussed in the Sec.3.1. Due to the relatively slow thermal diffusion in metals, the shorter pulse or the later the peak heat flux, the maximum temperature becomes higher, and the erosion becomes larger. These tendencies were generally reproduced in the PIXY calculations with and without vapor shielding. Thus, this comparison gives a validity of the thermal calculations in PIXY.

In Fig. 3, when the vapor shielding is included, the erosion amount is reduced by several tens of percent in all pulse shapes. Further analysis on the erosion suppression by the vapor shielding is in the next section using Fig. 5 .

\subsection{Vapor shielding efficiencies for different pulse shapes}

Rate of erosion suppression by the vapor shielding is defined as $\left(\delta_{\text {no-shield }}-\delta_{\text {shield }}\right) / \delta_{\text {no shield }}$, where $\delta$ is the erosion amount. The rate of erosion suppression is plotted in Fig. 5. For all pulse shapes, the erosion suppression of the Be wall show peaks at $1.5 \mathrm{MJ} / \mathrm{m}^{2}-2 \mathrm{MJ} / \mathrm{m}^{2}$. The peak can be explained as follows. A certain amount of energy density is necessary for the vapor emission. The vapor shielding is weak at low energy density pulses. However, the vapor shielding effect is finite, and it cannot suppress all the heat flux. Thus, when the energy density becomes higher, erosion caused by the plasma cannot be fully shielded by vapor. Comparing each pulse shape, the "Negative Ramp" has the highest shielding efficiency among the triangular shapes. This can be inferred from Fig. 2. The initial peak heat flux of the "Negative Ramp" causes a significant temperature increase and vapor emission. The emitted vapor can efficiently shield the subsequent heat flux which is lower than the initial peak heat flux. If the peak heat flux occurs later, the timing of vapor emission delays and shielding performance is limited as appears for the "Positive Ramp" cases.

It is predicted that the transient heat pulse shape in fusion reactors will be similar to the "Negative Ramp". According to this study, the erosion caused by the "Negative Ramp" is lower than the square pulse shapes (Half Width Full Maximum). This reduced erosion has already been pointed out by $\mathrm{Yu}$ [24]. In the present study, we found further erosion suppression by the vapor shielding. The predicted erosion during the transient event can be further reduced if we consider the higher vapor shielding performance of the "Negative Ramp" pulse shape.

\section{Summary}

We examined vapor shielding efficiency of the Be wall under transient heat loads at different pulse shapes. The PIC-based vapor shielding simulation code, PIXY, successfully produced transient pulses with different shapes. Depending on the pulse shape, the maximum temperature changes for the same energy densities without vapor shielding. It was found for the first time that the effect of vapor shielding also changes for the pulse shapes. In the Negative Ramp shape, expected for disruptions, the vapor generated by the initial peak heat flux shows an effective shielding effect. It was shown that the actual amounts of erosions will be smaller compared to the conventional estimations of the erosions assuming square pulse shapes. 


\section{Acknowledgments}

This work was partially supported by JSPS KAKENHI Grant Number 17H01367, the NIFS Collaboration Research program (NIFS18KEMF134), the Japan Society of the Promotion of Science (JSPS) Coreto-Core Program JPJSCCA2019002, and Joint Special Design Team Collaborative Research Program (30K054).

[1] R.A. Pitts et al., J. Nucl. Mater. 438, S48 (2013).

[2] R.A. Pitts et al., Nucl. Mater. Energy 20, 100696 (2019).

[3] K. Ibano et al., Contrib. Plasma Phys. 56, 705 (2016).

[4] K. Krieger et al., Nucl. Fusion 58, 026024 (2018).

[5] J.W. Coenen et al., Nucl. Fusion 55, 023010 (2015).

[6] Y. Hamaji et al., Plasma Fusion Res. 11, 2405089 (2016).

[7] Y. Hamaji et al., Nucl. Mater. Energy 12, 1303 (2017).

[8] J. Linke et al., Nucl. Fusion 51, 073017 (2011).

[9] N.S. Klimov et al., Nucl. Mater. Energy 12, 433 (2017).
[10] Y. Kikuchi et al., J. Nucl. Mater. 463, 206 (2015).

[11] Y. Kikuchi et al., J. Nucl. Mater. 438, S715 (2013).

[12] G. Pintsuk et al., Fusion Eng. Des. 82, 1720 (2007).

[13] J.H. Yu et al., Nucl. Fusion 55, 093027 (2015).

[14] D. Motoi et al., Fusion Eng. Des. 165, 112209 (2021).

[15] A.A. Pshenov et al., Phys. Procedia 71, 14 (2015).

[16] I. Sakuma et al., J. Nucl. Mater. 463, 233 (2015).

[17] H. Wurz et al., J. Nucl. Mater. 233, 798 (1996).

[18] K. Ibano et al., Nucl. Fusion 59, 076001 (2019).

[19] K. Ibano et al., Contrib. Plasma Phys. 58, 594 (2018).

[20] R.J. Hawryluk et al., Nucl. Fusion 49, 065012 (2009).

[21] A. Tanaka et al., Contrib. Plasma Phys. 58, 451 (2018).

[22] T. Takizuka and H. Abe, J. Comput. Phys. 25, 205 (1977).

[23] H.P. Summers and M.G. O'Mullane, in AIP Conference Proceedings ATOMIC AND MOLECULAR DATA AND THEIR APPLICATIONS (ICAMDATA-2010), (2011) 179-187.

[24] J.H. Yu et al., Nucl. Fusion 55, 093027 (2015).

[25] J. Coburn et al., Nucl. Mater. Energy 28, 101016 (2021). 